\title{
A Design of Resistance Optimization System for Unmanned Submersible Vehicle Based on Response Surface Method
}

\author{
Zi-Fan WEl ${ }^{1, a}$, Meng-Yun WANG ${ }^{2, b}$, Qiang YU ${ }^{3, c}$, Song-Lin YANG $^{4, d}$ \\ 1,2,3,4 Jiangsu University of Science and Technology, Zhenjiang,China,212003 \\ ajkdwzf@163.com, bhinnykitty@163.com, cjsslsxyq@163.com, dysl560516@vip.163.com
}

${ }^{*}$ Corresponding author

\begin{abstract}
Keywords: Unmanned Submersible Vehicle, Response Surface Method(RSM), Resistance Optimization System.
\end{abstract}

\begin{abstract}
Estimating the sailing resistance of unmanned submersible vehicle when navigating in the water is an important part for designing the hull profile. As there is no wave resistance when the unmanned submersible vehicle navigating in the water, so the main hull resistance mainly includes friction resistance and viscous pressure resistance. There are a lot factors affecting the resistance of the hull, including the length of bow,the length of stern, the length of middle body, the molded breadth, the molded depth, the design speed and the aspect ratio of rudder. The above factors have been selected as design variables of the resistance optimization system and the regression relationship between design variables and the frictional resistance viscous pressure resistance has been established by the response surface method, which can obtain the approximate function formula between the objective function, constraints and the design variables, providing a basis for the establishing of resistance optimization system. The design can be expanded to an overall situation by this method, then this complex disciplinary analysis can be separated from the optimization process, which can reduce the amount of calculation and optimization time. The application of polynomial response surface method in resistance optimization system has been studied in this paper and the accuracy of five polynomial response surface functions also have been studied, which reflected that the accuracy of second-order response surface is better than that of the first-order and third-order response surface. The response surface of wet area of the hull have a high fitting accuracy.
\end{abstract}

\section{Introduction}

Response Surface Method, which is called RSM for short, is a approximation method of system response, based on multiple fitting ideas[1]. Precise relationship between the design and dependent variables can be obtained by this method with few test points, which can be expressed by a simple algebraic relationship. Experimental design theory was adopted in response surface method in the design space for testing the given set of design points to get a response surface model of the objective function, which can predict the response value of non- pilot points[2]. Therefore, when dealing with the analysis of multi-factors, the regression relationship between a dependent variable and multiple independent variables can be obtained by this method[3].

Currently, the neural network and polynomial response surface methods are the more commonly used global approximation methods[4]. The basic concept is that, the numerical model is replaced by a polynomial with a clear expression of form to reflect the approximate relationship between the objective function and the design variables. This method can been used in the optimized design[5][6], which can solve that how to establish the approximate function between the objective function, constraints and design variables through reasonable design of experiment.

In this paper, the AUV of AUTOSUB is the research object. Using multiple response surface equations and the database of CFD numerical results, the design of resistance optimization system has been established. Finally, five kinds of different response surface equations of wet area of hull, the viscous pressure resistance and friction resistance have been established to study the accuracy of the five kinds of response surface polynomial functions. 


\section{Polynomial Response Surface}

As the relationship between the dependent and independent variables is unknown, so choosing what kind of function formula will largely affect the fitting accuracy of the response surface. Generally, two requirements should be considered. On one hand, on the precondition of that the mathematical expression can describe the real function, the mathematical expression of response surface function should be as simple as possible. On the other hand, when designing the response surface function, the number of undetermined coefficients should be minimized, in order to reduce the workload of physical experiments or numerical analysis[7][8]. The common forms of polynomial functions of response surface are showed as follows.

linear equation:

$$
\tilde{y}=\alpha_{0}+\sum_{j=1}^{n} \alpha_{j} x_{j}
$$

Quadratic equation without cross-terms

$$
\tilde{y}=\alpha_{0}+\sum_{j=1}^{n} \alpha_{j} x_{j}+\sum_{j=1}^{n} \alpha_{i j} x_{j}^{2}
$$

Quadratic equation with cross-terms

$$
\tilde{y}=\alpha_{0}+\sum_{j=1}^{n} \alpha_{j} x_{j}+\sum_{i=j+1}^{n} \sum_{j=1}^{n} \alpha_{i j} x_{i} x_{j}+\sum_{j=1}^{n} \alpha_{j j} x_{j}^{2}
$$

Cubic equation without cross-terms

$$
\widetilde{y}=\alpha_{0}+\sum_{j=1}^{n} \alpha_{j} x_{j}+\sum_{j=1}^{n} \alpha_{j j} x_{j}^{2}+\sum_{j=1}^{n} \alpha_{j j j} x_{j}^{3}
$$

Cubic equation with cross-terms

$$
\tilde{y}=\alpha_{0}+\sum_{j=1}^{n} \alpha_{j} x_{j}+\sum_{i=j}^{n} \sum_{j=1}^{n} \alpha_{i j} x_{i} x_{j}+\sum_{j=1}^{n} \alpha_{j j} x_{j}^{2}+\sum_{j=1}^{n} \alpha_{j j j} x_{j}^{2}+\sum_{j \neq i}^{n} \sum_{i=1}^{n} \alpha_{i i j} x_{i}^{2} x_{j}+\sum_{k=j+1}^{n} \sum_{i=j+1}^{n} \sum_{j=1}^{n} \alpha_{i j k} x_{i} x_{j} x_{k}
$$

The equations above can be expressed into unified form, which has been shown as follows.

$$
\tilde{y}=\sum_{j=0}^{k-1} \alpha_{j} x_{j}
$$

Where $\mathrm{k}$ is the number of undetermined coefficients; $\alpha$ is the uniform coefficient; $\tilde{y}$ is dependent variable; $\mathrm{x}$ is independent variable;

Tab. 1 Undetermined Coefficients of Different Response Surface Function

\begin{tabular}{cc}
\hline The form of function & The number of undetermined coefficients \\
\hline linear equation & $\mathrm{n}+1$ \\
$2 \mathrm{n}+1$ \\
$(\mathrm{n}+1)(\mathrm{n}+2) / 2$ \\
Quadratic equation without cross-terms & $(\mathrm{n} 2+5 \mathrm{n}+2) / 2$ \\
Quadratic equation with cross-terms & $(3 \mathrm{n} 2+3 \mathrm{n}+2) / 2(\mathrm{n}=2),(\mathrm{n} 3+3 \mathrm{n} 2+14 \mathrm{n}+6) / 6(\mathrm{n}>2)$ \\
Cubic equation without cross-terms & \\
Cubic equation with cross-terms &
\end{tabular}

To determine the coefficient $\alpha, \mathrm{m}$ times $(\mathrm{m} \geqslant \mathrm{k}$ ) independent tests should be done, and each test should has different values, which can get a response value corresponding to $\mathrm{m}$ sample points, and the response surface matrix has been established as follows:

$$
X B-Y=E
$$

Where $\mathrm{X}$ is the matrix of variable values; $\mathrm{Y}$ is the matrix of response values; $\mathrm{B}$ is the matrix of coefficients; $\mathrm{E}$ is the error matrix; 
The least squares method and the theory of matrix analysis are used to minimize the system error.

$$
\begin{aligned}
& S(\beta)=\|E\|^{2}=(X B-Y)^{T}(X B-Y) \rightarrow \min \\
& \nabla S(\beta)=\nabla\left((X B-Y)^{T}(X B-Y)\right)=2(X B-Y)^{T} X=0 \\
& B=\left(X^{T} X\right)^{-1} X^{T} Y
\end{aligned}
$$

The accuracy of the response surface can be expressed by correlation coefficient, corrected correlation coefficient and the root mean square error.

$$
\begin{aligned}
& R^{2}=1-\frac{S S_{E}}{S S_{T}} \quad R_{a}^{2}=1-\frac{S S_{E} /(n-m)}{S S_{T} /(n-1)} \quad R S M E=\sqrt{S S_{E} /(n-m)} \\
& S S_{T}=\sum_{i=1}^{n}\left(y_{i}-\bar{y}\right)^{2} \quad S S_{E}=\sum_{i=1}^{n}\left(y_{i}-\hat{y}\right)^{2}
\end{aligned}
$$

where $\mathrm{R}^{2}$ is the correlation coefficient; $R_{a}^{2}$ is the corrected correlation coefficient; RMSE is the root mean square error.

The correlation coefficient is a value changing between 0 to 1 , which is closer to 1 , the error value is smaller, the more accurate fitting equation is, and with the increase of the number of independent variables, the value of correlation coefficient increases. Considering the influence of the number of parameters, when the number of parameters increase the corrected correlation coefficient not necessarily increases, so, the accuracy of the fitting equations of different number of parameters can be compared by the corrected correlation coefficient. The root mean square error is an unbiased estimate of response equation, the smaller the value of the root mean square error is, the higher the accuracy of the response surface is.

\section{The Design of Resistance Optimization System of Submersible Vehicle}

In this paper, the AUV of AUTOSUB is the research object. Considering factors that affect the resistance of the hull navigating in the water, sixteen geometric models have been constructed and numerically simulated by CFD, which created the database of navigating resistance. The response surface equations of wet area of hull, the volume of hull and the viscous pressure resistance have been established to establish the resistance optimization system of the AUV. The flow chart of the resistance optimization system for Unmanned Submersible Vehicle has been shown in Fig.1. And the specific process of establishing the resistance optimization system has been shown as follows.

Friction resistance :

$$
\begin{aligned}
& R_{f}=\left(C_{f}+\Delta C_{f}\right) \frac{1}{2} \rho V_{s}^{2} S \\
& C_{f}=\frac{0.075}{(\log \operatorname{Re}-2)^{2}}
\end{aligned}
$$

Where Re is Reynolds Number; $\rho$ is the density of water; $\mathrm{S}$ is the wet area of the hull; Vs is the velocity of the AUV; $\Delta C_{f}$ is the additional roughness coefficient; $C_{f}$ is coefficient of resistance;

1)The response surface of wet area of hull:

$S / D^{2}=f_{1}\left(L_{h} / D, L_{\mathbf{m}} / D, L_{a} / D, \lambda\right)$

2)The response surface of the volume of hull:

$$
\nabla / D^{3}=f_{2}\left(L_{h} / D, L_{\mathbf{m}} / D, L_{a} / D, \lambda\right)
$$


3)The response surface of the viscous pressure resistance:

$$
R_{p v} / \Delta=f_{3}\left(L_{h} / D, L_{m} / D, L_{a} / D, \log \mathrm{Re}\right)
$$

Where $\mathrm{L}_{h}$ is the length of bow; $\mathrm{L}_{m}$ is the length of middle body; $\mathrm{L}_{\mathrm{a}}$ is the length of stern; $\mathrm{D}$ is the average value of molded breadth and molded depth; $\lambda$ is the aspect ratio of the rudder; $R e$ is the reynolds number;

4) Design variables of the resistance optimization system are the length of bow, the length of middle body, the length of stern, the molded breadth, the molded depth, the aspect ratio of the rudder and the velocity of ship.

5) Admiralty coefficient has been selected as the objective function, which is shown as follows.

$$
C_{s p}=\frac{V_{s}^{3} \Delta^{2 / 3}}{P_{s}}=\frac{V_{s}^{2} \Delta^{2 / 3} \eta_{R} \eta_{0} \eta_{s} \eta_{H}}{R_{t}}
$$

6) Constraints Conditions:

Balance between buoyancy and displaced weight:

$$
\nabla=L B H C_{B}
$$

Balance between effective thrust and resistance:

$$
N_{p} K_{T} \rho N^{2} D_{P}^{4}(1-t)=R_{t}
$$

Balance between torque received by screw from main engine and torque from hydrodynamic resistance:

$$
\frac{\eta_{R} \eta_{s} P_{s}}{2 \pi N}=K_{Q} \rho N^{2} D_{P}^{5}
$$

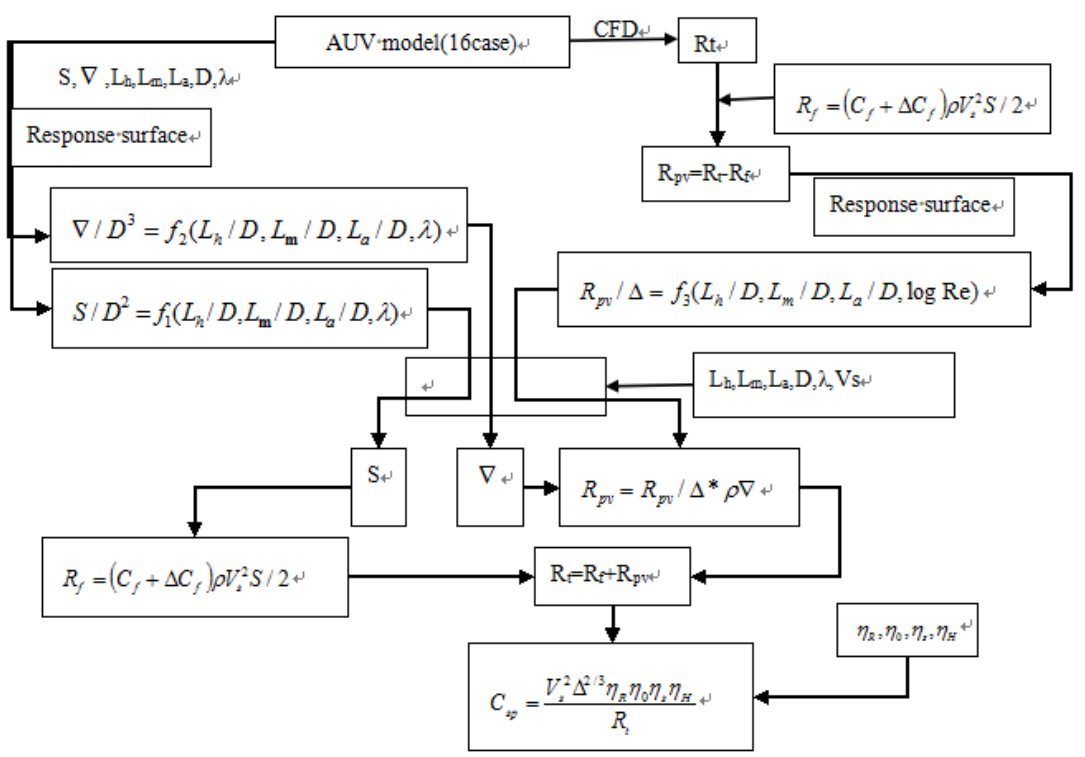

Fig. 1The Flow Chart of the Resistance Optimization System for Unmanned Submersible Vehicle

\section{Accuracy Analysis of Response Surface}

Through analyzing different response surface equations, including the response surface of wet area of hull, the response surface of viscous pressure resistance and the response surface of friction resistance, which use the AUTOSUB as the research object, to study the accuracy of five kinds of functions of response surface including linear equation, quadratic equation without cross-terms, quadratic equation with cross-terms, cubic equation without cross-terms and cubic equation with cross-terms. 


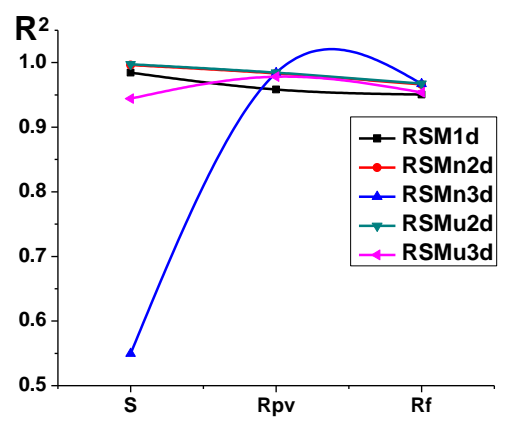

Fig.2 The Correlation Coefficient of the Five Kinds of Polynomial Function for Three Different Response Surfaces

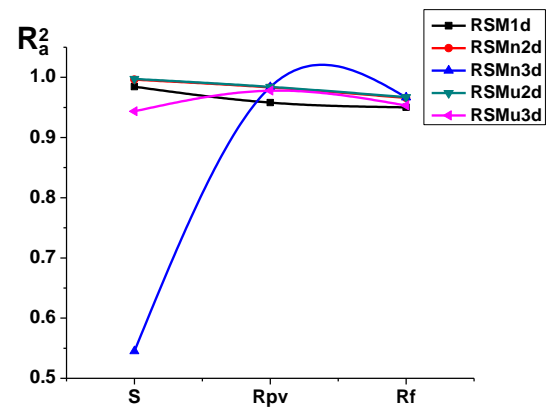

Fig.3 The Corrected Correlation Coefficient of the Five Kinds of Polynomial Function for Three Different Response Surfaces

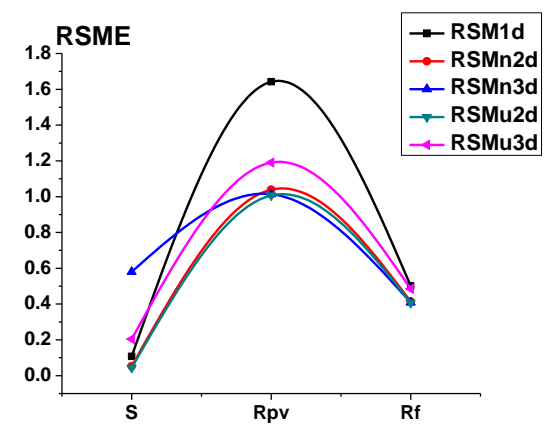

Fig.4 The Root Mean Square Error of the Five Kinds of Polynomial Function for Three Different Response Surfaces

As can be seen from the figures above including Fig.2, Fig.3 and Fig.4, the quadratic equations with cross-terms have the highest correlation coefficient and the highest corrected correlation coefficient, the lowest root mean square error. So, the response surface of the quadratic equations with cross-terms has the highest accuracy. The cubic equations without cross-terms have the lowest correlation coefficient and the lowest corrected correlation coefficient, which reflects its low fitting accuracy.

Comparing the response surface equations of wet area of hull, the quadratic equations with cross-terms has the highest accuracy, but the cubic equations without cross-terms has the lowest accuracy. In addition, comparing the response surface equations of viscous pressure resistance and friction resistance, the quadratic equations with cross-terms has the highest accuracy, but the linear equation has the lowest accuracy. What's more, the quadratic equation without cross-terms has the best fitting accuracy except the quadratic equation with cross-terms, so, the response surface of quadratic equation has the best fitting accuracy. 


\section{Conclusion}

In this paper, the AUV of AUTOSUB is the research object. The resistance optimization system has been established through constructing the response surfaces of wet area of hull, volume of hull and the viscous pressure resistance. Finally, five kinds of different response surface equations of wet area of hull, the viscous pressure resistance and friction resistance have been established to study the accuracy of the five kinds of response surface polynomial functions using the correlation coefficient, the corrected correlation coefficient and the root mean square error as standards. Studies have shown that the response surface of quadratic equation has the best fitting accuracy and the response surface can be used to construct the resistance optimization system, which can simplify the whole optimization system and providing a design concept for the further optimization design.

\section{Acknowledgement}

The Basic Scientific Research Project of Ministry of Industry and Information Technology of the People's Republic of China: project number: A2420110001.

\section{References}

[1]Sui Yunkang, Yu Hui Ping. The improvement of response surface method and its application in engineering optimization. Beijing: Science Press, 2011.02.

[2]MYERS R H;MONTGOMERY D C Response surface methodology: process and product optimization using designed experiments 2002 .

\section{[3]Liang Jun. SHIP HULL LINES OPTIMIZATION BASED ON.}

HYDRODYNAMIC PERFORMANCE. master thesis of Jiaotong University of Shanghai. 2008: 21 -25 .

[4]Qian Jiankui, Mao Xiao Fei, Wang Xiaoyi. Ship hull automated optimization of minimum resistance via CFD and RSM technique. Ship mechanics. 2012,16 (1): 1-8.

[5]Deng Lei, Qiang Zhide, Song Wenping, etc. Multi-Objective Aerodynamic Optimization Design of Wind Turbine Blade based on Response Surface Methodology [J]. Aerodynamics Sinica, 2012, 30 (3): $1-9$.

[6]Cheng Ye, Zhou Cuiying, Huang Linchong, etc. Multi-dimensional exponential interpolation response surface method for reliability analysis of complex structural engineering [J]. Journal of ShenZhen university science and engineering, 2012, 29(5): 1-6.

[7]Shi Yanyan, Sun Zhili, Yan Ming. Application of Response Surface Methodology in Sensitivity Calculation of Reliability [J].JOURNAL OF NORTHEASTERN UNIVERSITY(NATURAL SCIENCE), 2009, 30 (2): 1 -5.

[8]Yan Ming, Sun Zhili, Yang Qiang,etc. ANALYSIS METHOD OF RELIABILITY SENSITIVITY BASED ON RESPONSE SURFACE METHODS [J]. CHINESE JOURNAL OF MECHANICAL ENGINEERING, 2007, 43(10): 1-7. 